Anton Liebetrau

\title{
TopSpeed Modula-2 Units und Utilities
}

Die professionelle Toolbox

für den Softwareentwickler

mit über 200 Routinen 


\section{Numerik-Praktikum mit VISU}

Das umfangreiche Softwarepaket

zur Visualisierung Numerischer Mathematik

für Mathematiker, Naturwissenschaftler, Ingenieure

von Rolf Schröder

\section{Vieweg GraphikManager: ARA}

Ein kompaktes speicherresidentes Graphikprogramm

für die EGA-Graphikkarte

von Markus Weber

\section{Wissensverarbeitung mit DEDUC}

Ein Expertensystemshell mit Benutzeranleitung sowie einem Lehrbuch zur Wissensverarbeitung, Folgenabschätzung und Konsequenzenbewertung von Hartmut Bossel, Bernd R. Hornung und Karl-Friedrich Müller-Reißmann

\section{TopSpeed Modula-2 Units und Utilities}

Die professionelle Toolbox für den Softwareentwickler mit über 200 Routinen

von Anton Liebetrau

\section{Vieweg DecisionManager}

Ein Programmpaket zur Lösung linearer Probleme mit mehreren Zielfunktionen

von Jürgen Hansohm und Michael Hänle

\section{Vieweg ProjectManager}

Software zum modernen Projektmanagement mit Benutzerhandbuch von Erik Wischnewski 
Anton Liebetrau

\section{TopSpeed Modula-2 Units und Utilities}

Die professionelle Toolbox für den Softwareentwickler mit über $\mathbf{2 0 0}$ Routinen 
Die Software-Routinen, beschrieben in diesem Buch, dürfen in eigenen Anwendungsprogrammen verwendet werden. Der Programmierer darf eigene Programme, die diese Routinen enthalten, in ausführbarer und kompilierter Form ohne Einschränkungen oder Verpflichtungen zu zusätzlichen Lizenzgebühren weiterverkaufen; dasselbe gilt für die Wörterbuch-Datei SPELL.LEX, wenn diese in eigenen Programmen verwendet wird. Die Quellcodes der einzelnen Module oder nur Teile davon dürfen weder weitergegeben noch verkauft werden.

Die zu diesem Buch gehörenden Demo-Programme (SPELLCHK.MOD und MINIEDIT.MOD) dürfen nicht weiterverkauft werden, jedoch können beliebige Teile der Demo-Programme in eigenen Programmen verwendet werden. Diese neuen Anwendungen müssen sich jedoch bei einem Verkauf so weitgehend von SPELLCHK.MOD und MINIEDIT.MOD unterscheiden, daß sie im Sinne des Urheberrechtes als eigenständige Programme schützbar sind.

Das in diesem Buch enthaltene Programm-Material ist mit keiner Verpflichtung oder Garantie irgendeiner Art verbunden. Der Autor und der Verlag übernehmen infolgedessen keine Verantwortung und werden keine daraus folgende oder sonstige Haftung übernehmen, die auf irgendeine Art aus der Benutzung dieses Programm-Materials oder Teilen davon entsteht.

Alle Rechte vorbehalten

(c) Springer Fachmedien Wiesbaden 1991

Ursprünglich erschienen bei Friedr. Vieweg \& Sohn Verlagsgesellschaft mbH, Braunschweig 1991

Softcover reprint of the hardcover 1st edition 1991

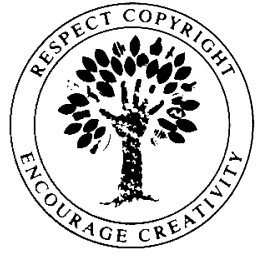

Das Werk einschließlich aller seiner Teile ist urheberrechtlich geschützt. Jede Verwertung außerhalb der engen Grenzen des Urheberrechtsgesetzes ist ohne Zustimmung des Verlags unzulässig und strafbar. Das gilt insbesondere für Vervielfältigungen, Übersetzungen, Mikroverfilmungen und die Einspeicherung und Verarbeitung in elektronischen Systemen.

Umschlagsgestaltung: Ludwig Markgraf, Wiesbaden

ISBN 978-3-528-04796-2

ISBN 978-3-322-89472-4 (eBook)

DOI 10.1007/978-3-322-89472-4 


\section{Vorwort}

Seit TopSpeed Modula-2 auf dem Software-Markt erhältlich ist, hat die moderne Programmiersprache Modula-2 unter den Software-Entwicklern sichtlich an Beliebtheit gewonnen. Dies läßt sich einerseits daran erkennen, daß vermehrt Bücher zu dieser Programmiersprache auf dem Markt erscheinen und andererseits in den einschlägigen Computer-Zeitschriften Quellcodes in TopSpeed Modula-2 abgedruckt werden. Dazu beigetragen haben die komfortable Entwicklungsumgebung von TopSpeed Modula-2, die ausgezeichneten Bibliotheks-Module (Prozeß-Verwalter, Fenstertechnik ...), der schnelle Compiler, der intelligente Linker, der kompakte und optimierte Programmcode und nicht zuletzt der günstige Preis des gesamten Entwicklungs-Systems.

Dieses Buch eignet sich besonders für denjenigen Programmierer, der für seine Software-Entwicklungen TopSpeed Modula-2 (Versionen 1.xx und 2.xx) verwendet und trotz der rund 300 Prozeduren und Funktionen an Grenzen stößt.

Die zehn in diesem Buch beschriebenen Module enthalten mehr als 200 neue Routinen und erweitern TopSpeed Modula-2 beträchtlich. Es enthält Routinen zur Textverarbeitung und Maussteuerung, erleichtert das Verwalten von Meldungen (die mit dem komfortablen Meldungs-Editor MSGEDIT.EXE geschrieben werden) und Generieren von Menüs. Besonders nennenswert erscheint mir das Modul Spell; mit ihm wird es möglich, anhand bis zu zehn gleichzeitig geöffneten Referenz-Wörterbüchern (fünf verschiedene Größen möglich) Orthographie-Fehler in einem Text zu suchen. Es erkennt zusammengesetzte Hauptwörter (mit und ohne Fugen-s), unterscheidet zwischen Groß- und Kleinschreibung und ist außerdem unglaublich schnell. Ein ähnliches Modul ist bis zum heutigen Zeitpunkt meines Wissens nicht auf dem Software-Markt erhältlich.

Dieses Buch ist in zwei Teile gegliedert. Der erste Teil beschreibt die Wirkungsweise der einzelnen Routinen und verdeutlicht einzelne Aspekte durch kurze Beispiel-Programme. Im zweiten Teil (Anhang) finden Sie neben nützlichen Tabellen und einer Übersicht aller Module auch eine ausführliche Beschreibung der beiden umfangreichen Demo-Programme SPELLCHK.MOD und MINIEDIT.MOD, die auf einer der beiliegenden Disketten enthalten sind. Schließlich werden die vom Modul Spell verwendeten Algorithmen und Datenstrukturen ausführlich erläutert und mit Graphiken verdeutlicht.

Die einzelnen Module sind mit größter Sorgfalt geschrieben und getestet worden. Da sich aber Fehler nie ganz ausschließen lassen oder vielleicht einzelne Routinen nicht Thren Vorstellungen entsprechen, bin ich für Hinweise und Anregungen jederzeit dankbar. 


\section{Inhaltsverzeichnis}

Vorwort ............................................................. V

Beschreibung der Module ................................................... 1

Das Modul DOS ........................................................ 2

Das Modul Key .......................................................... 34

Das Modul Menu ..................................................................... 38

Das Modul Message ........................................................ 59

Das Modul Mouse .......................................................... 74

Das Modul RuntimeCheck ........................................................ 119

Das Modul Spell ............................................................. 124

Das Modul Sys ........................................................... 145

Das Modul Txt ........................................................ 173

Das Modul Workbox ........................................................ 200

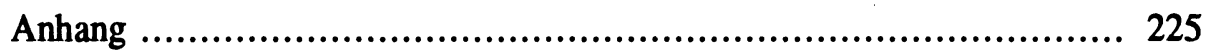

A - Erläuterungen zum Modul Spell .................................... 226

B - Übersicht der Module ............................................. 236

C - Demo-Programme .................................................... 245

D - Inhalt der beiliegenden Disketten .................................. 253

E - Tastencodes ........................................................ 255

F - Graphikzeichen für Tabellen ..................................... 258

G - IBM-Zeichensatz ............................................... 259

Literaturhinweise ............................................................ 261

Sachwortverzeichnis ..................................................... 262 